Journal of Engineering and Applied Sciences 14 (1): 164-169, 2019

ISSN: 1816-949X

(C) Medwell Journals, 2019

\title{
Data Extracted and Fitting Which is Calculated from Laser Spot Imaging Passes Through Multi Media
}

\author{
Zahraa Saad Mohsen, Mutasim Ibrahim Malik and Ali Kamel \\ Department of Physics, College of Science, University of Wasit, Kut, Iraq
}

\begin{abstract}
This research attempts to study the behavior of the Helium-Neon laser spot by using a digital camera and passing through various physical media (distilled water, water of the Tigris River of Iraq-Wasit) to study its properties and develop equations that describe this behavior for several readings by calibrating them with TDS device. The equation by which a system can predict the behavior of a laser spot is calculated depend on the changing of the intensity of the resulting laser spot where a relationship is found between the intensity of the laser spot and the amount of concentrations of dissolved molecules in liquids and calculating the deposition time of the suspended materials. The final results showed the linear relationship between deposition time and intensity as well as deposition time and the readings of the TDS water system of the Tigris River, the results showed that it is possible to study the behavior of the laser spot when passing through different media and to find a mathematical relationship to describe and predict the results. This behavior is described by studying the intensity with concentrations of different materials and within different media. The deposition time was also calculated for different media based on the same principle. This was done by a system were build and a multiple software's such as ImageJ, Origin Lab 9.0, MATLAB R2013b and CurveExpert Professional 2.6.5.
\end{abstract}

Key words: Laser behavior, image processing, curve fitting, attenuation, deposition, concentration

\section{INTRODUCTION}

The laser beams sources emit beams of electromagnetic radiation that have the same nature as the rays emitted from conventional optical sources but are highly characterized by high luminance and purity of color with the associated optical beams. Over the past half century, laser equipment has been developed where it is possible to obtain these rays at a wide range of wavelengths or frequencies covered from the infrared ray area and may reach the X-ray area. From this point of view, it was found that when lasers interact with different physical media, this reaction takes a different approach than that emitted from traditional sources when interacting with the material media (Al-Rogaibah, 2009). When a beam of light is incident on material medium and propagate through it, various light-mater interaction phenomenon takes place. These interactions can occur at the surface or inside the medium components and the rest of light may penetrate the medium (transmission). Due to this interaction of light with the medium components there might be reflection on the surface and attenuation due to scattering and absorption inside the medium by internal suspended components (Allmen and Blatter, 2013). The absorption properties are strongly dependent on the wave length of the incident light while reflection and scattering have negligible effect on wave length of incident light.
Interaction of light with material medium depends on the composition and density of the medium and the wavelength and energy of the light (Al-Azzawi and Abdulzahra, 2014). In any conditions of material medium, when light tries to propagate through the given medium the following properties of light can happen: transmission, absorption, scattering. Al-Azzawi and Abdulzahra (2014) studied the visual characteristics of tap water using a helium-neon laser beam with different power and some samples with different densities. These samples contain sodium chloride salts and different concentrations of clay. When we increase the concentration and length of the samples, we observe the decrease in transmittance and the absorption and attenuation coefficients while increasing the absorption. Absorption and absorption coefficient for tap water is greater than sodium chloride salt and less than the gluten alone and when the mixture is mixed with salt (the length of the sample is constant) (Jassim, 2014; Zielesny, 2016) conducted an analytical study of laser communication in the Shatt Al-Hilla water using a laser source at different wavelengths $(407,473$, 532,632 and $810 \mathrm{~nm}$ ). It is transmitted through samples of water with different turbidity (pure and turbid) high turbidity). The blue laser light began to function as carrier signal and for this reason it can proceed underwater with the lowest absorption factor $30 \mathrm{~cm}^{-1}$ and the maximum transmission distance is $78 \mathrm{~m}$ (Hansen et al., 2013).

Corresponding Author: Zahraa Saad Mohsen, Department of Physics, College of Science, University of Wasit, Kut, Iraq 
Curve fitting: Curve fitting is process of finding equation to approximate straight line and curves that best fit given sets of data in our subject, we use the least square sum of the number method to find fit data (Banuelos-Cabral et al., 2017).

\section{Curve fitting methods}

Linear curve fitting: With this method, we compute the coefficients $\mathrm{m}$ (slope) and (y-intercept) of the straight-line equation:

$$
\mathrm{Y}=\mathrm{mx}+\mathrm{b}
$$

where the coefficients $\mathrm{m}$ and $\mathrm{b}$ are found from the following relations:

$$
\begin{aligned}
& \left(\sum \mathrm{x}^{2}\right) \mathrm{m}+\left(\sum \mathrm{x}\right) \mathrm{b}=\sum \mathrm{xy} \\
& \left(\sum \mathrm{x}\right) \mathrm{m}+\mathrm{nb}=\sum \mathrm{y}
\end{aligned}
$$

Where:

$\Sigma \mathrm{x}=$ Sum of the number $\mathrm{x}$

$\Sigma y=$ Sum of the number $y$

$\Sigma x y=$ Sum of the number of the product xy

$\Sigma \mathrm{x}^{2}=$ Sum of the number $\mathrm{x}$ squared

$\mathrm{n}=$ Number of data $\mathrm{x}$

With Carmer's rule, $m$ and $b$ are computed from :

$$
\begin{aligned}
& \mathrm{m}=\mathrm{D}_{1} / \Delta \quad \mathrm{b}=\mathrm{D}_{2} / \Delta \\
& \mathrm{D}_{2}=\left|\begin{array}{cc}
\sum \mathrm{x}^{2} & \sum \mathrm{xy} \\
\sum \mathrm{x} & \sum \mathrm{y}
\end{array}\right|, \quad \mathrm{D}_{1}=\left|\begin{array}{cc}
\sum \mathrm{xy} & \sum \mathrm{x} \\
\sum \mathrm{y} & \mathrm{n}
\end{array}\right| \begin{array}{cc}
\sum \mathrm{x}^{2} & \sum \mathrm{x} \\
\sum \mathrm{x} & \mathrm{n}
\end{array} \mid=\Delta
\end{aligned}
$$

Polynomial: Curved polynomial is a type of linear curve where the relationship between the variables $\mathrm{x}$ and $\mathrm{y}$ depends on the number of $\mathrm{k}$ multilevel rank. The polynomial curve represents a nonlinear relationship between the variable $\mathrm{x}$ and the mean of the corresponding $\mathrm{y}$ variable which is $\mathrm{E}(\mathrm{y}, \mathrm{x})$. It describes the nonlinear phenomenon. The curve shows the expected value of the $\mathrm{Y}$ variable by the $\mathrm{X}$ variables. In the linear curve, the model is said to be linear in case the variables are linear:

$$
\begin{aligned}
& Y=a_{0}+a_{1} x+\varepsilon \\
& Y=a_{0}+a_{1} x_{1}+a_{2} x_{2}+a_{11} x_{12}+a_{22} x_{22}+a_{12} x_{1} x_{2}+, \ldots,+\varepsilon
\end{aligned}
$$

is also a linear model. Of the polynomials in the second and the two variables, respectively. $\varepsilon$ be random error. Multidimensional models can be used in cases where the relationship between data and explanatory variables is curved.

Rational: $\mathrm{f}(\mathrm{x})$ is a function that called a rational function. It is a ratio of two polynomial functions. It written in the form:

$$
\mathrm{F}(\mathrm{x})=\mathrm{D}(\mathrm{x}) / \mathrm{H}(\mathrm{x})
$$

in which $\mathrm{D}$ and $\mathrm{H}$ are polynomial functions in $\mathrm{x}$ and $\mathrm{H}$ is not equal zero.

\section{MATERIALS AND METHODS}

System building: Building the system showed in Fig. 1. Set up a photographic system (digital camera) to the laser beam spot as it passes through the physical medium and takes multi frames for laser spot. Which passes through the physical medium.

The application of image data processing on the physical medium with the aim of extracting information from them. Several programs were used such as ImageJ, Origin Lab, MATLAB and curve expert.

\section{Devices and tools:}

- Source of helium-neon laser beam wavelength (632.8 $\mathrm{nm}$ )

- Glass container with dimensions $(75,50$ and $30 \mathrm{~cm})$ glass thickness $(6 \mathrm{~mm})$

- Digital Camera Nikon D3300

- A white screen or plate to show the pattern of laser spot

- TDS device (Total Dissolved Salts) to measure salinity ratio

- A sensitive electronic balance (Mettler Hk160) sensitive balance $\left(10^{-4} \mathrm{~g}\right)$ to measure salt weights

Procedure: The laser system, the glass container and the screen were installed on one straightness, so that, the distance between the source and the container and between the container and the screen was fixed along the experiment showed in Fig. 2. The camera angle was also fixed in the direction of the screen in a position where the best image of the spot was obtained.

The $40 \mathrm{~L}$ of Tigris water were prepared and take a picture every half hour for the spot of laser after passing through the container and it contains the river water. The TDS device measured the salinity every half an hour.

The images of the resulting laser spot were analyzed and the results obtained from the image processing

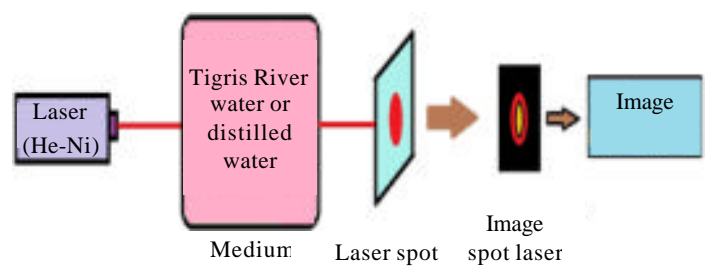

Fig. 1: Diagram for suggested work 


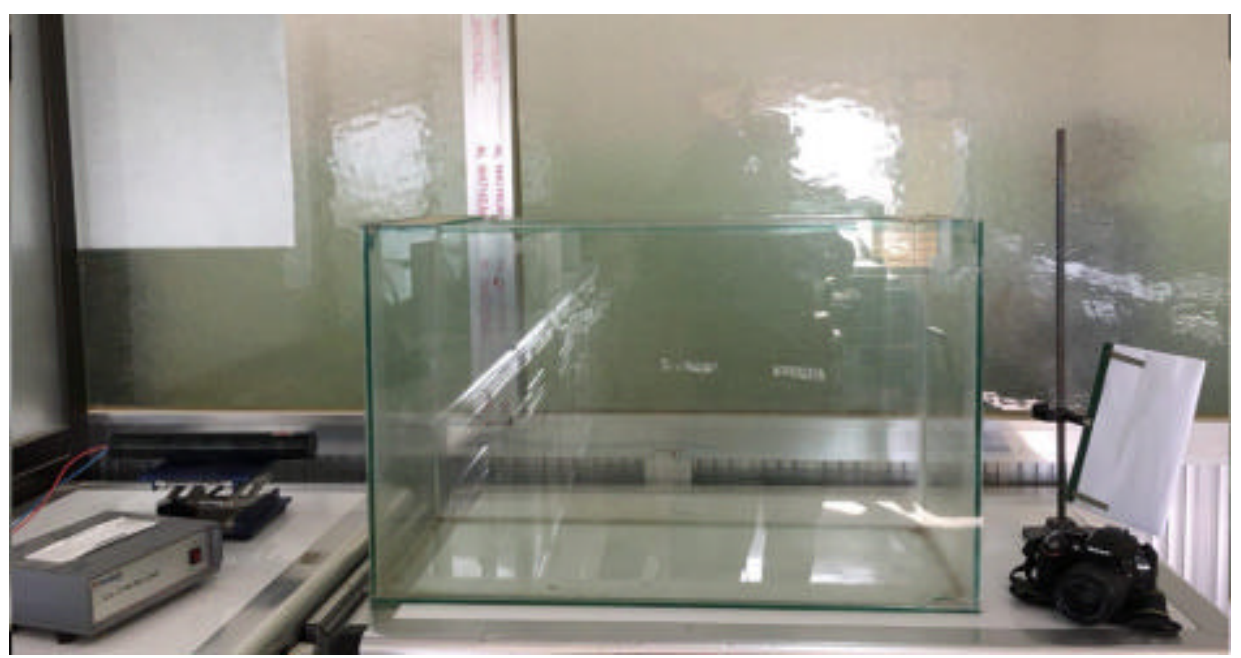

Fig. 2: System set up

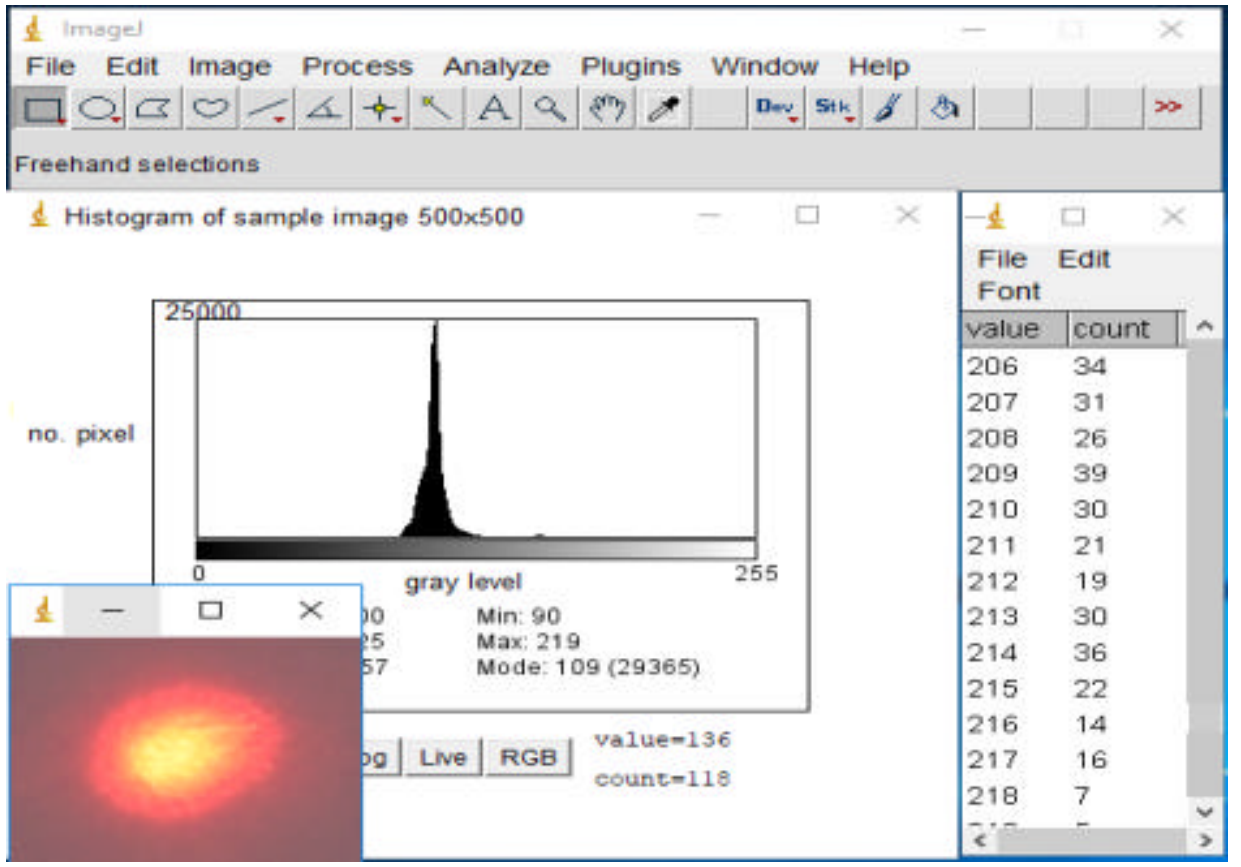

Fig. 3: Intensity calculated by histogram of sample image

technique to determine the intensity of the laser spot after passing through the river water. About $20 \mathrm{~L}$ of distilled water were prepared. And take a picture of the laser spot passing through the container after every $20 \mathrm{~g}$ of salt that is added to the water. The salt content was measured by TDS after each additional amount of salt.

The images of the resulting laser spot were analyzed and the results obtained from the image processing technology to determine the intensity of the laser spot after passing through the distilled water. The intensity is calculated by take average for maximum ten pixel from histogram for each image. As shown in Fig. 3 as example.

\section{RESULTS AND DISCUSSION}

\section{Tigris River water}

Fitting equation: By applied linear equation (Fig. 4): 


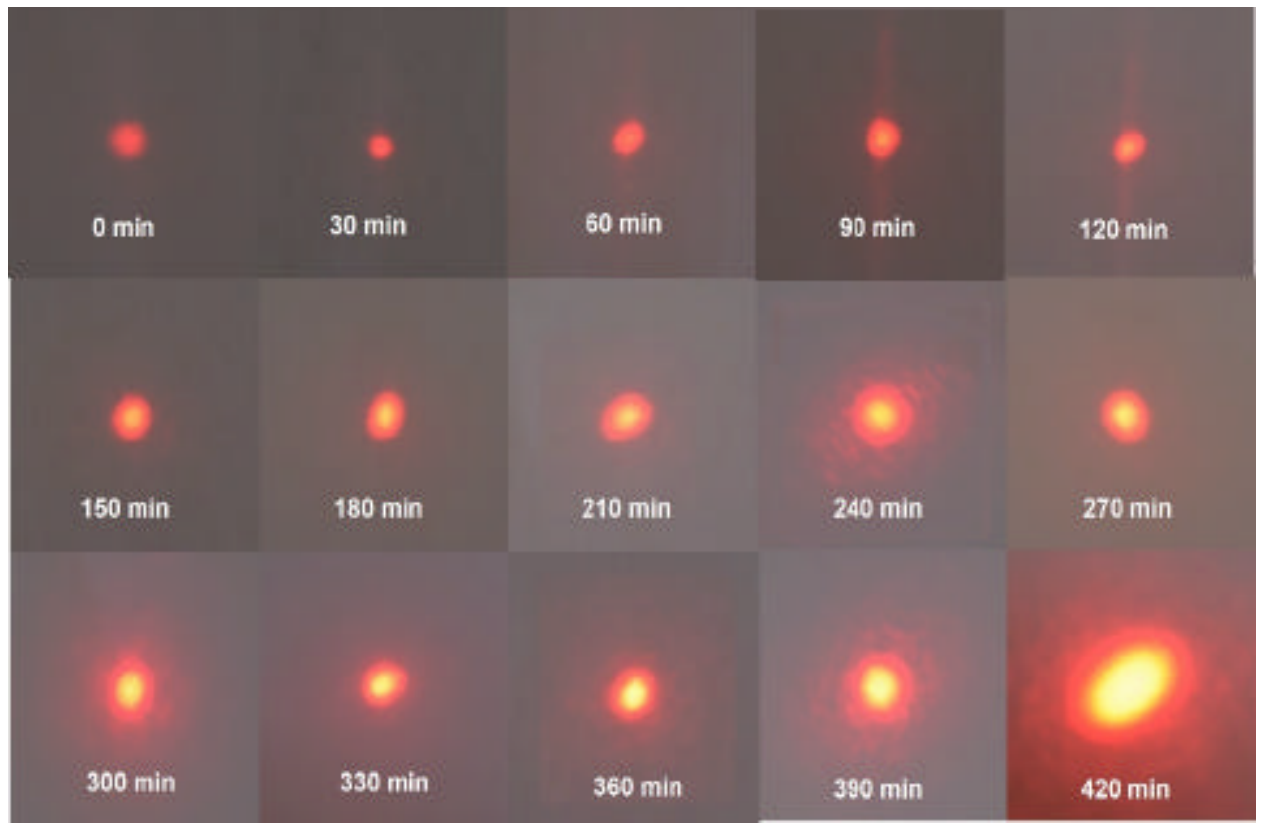

Fig. 4: Images of laser spots after passing through Tigris River water

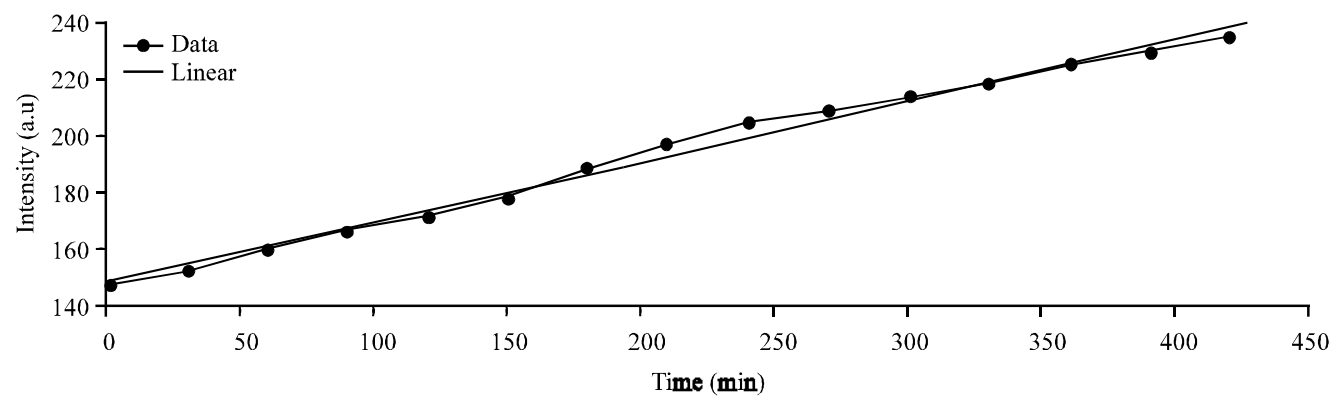

Fig. 5: Relationship between time of deposition and intensity

$$
\begin{aligned}
Y & =a+b x \\
(a & =148.333083, b=0.21411)
\end{aligned}
$$

Fitting equation: By applied linear equation (Table 1):

$$
\begin{aligned}
Y & =a+b x \\
(a & =946.4, b=-1.14476)
\end{aligned}
$$

\section{Distilled water}

Fitting equation: By applied rational equation:

$$
\begin{aligned}
& Y=(a+b x) /\left(1+c x+d x^{2}\right) \\
& a=254.984, b=123.737, c=0.57847, d=0.00705
\end{aligned}
$$

Fitting equation: By applied rational equation:

$$
\begin{aligned}
& Y=(a+b x) /\left(1+c x+d x^{2}\right) \\
& a=1022.859, b=398.653, c=-0.00973, d=0.006236
\end{aligned}
$$

There is a linear relationship between the time of deposition and the intensity of the laser beam showed in Fig. 5. The more time we observe, the greater the intensity due to the loss of the river's water, its kinetic energy where some of the suspended objects that it contains (Usually fine sand, salts and organic matter). These suspended objects cause scattering in the beam which acts as a dark barrier to the beam. Note that the intensity of the spot at the beginning of deposition is minimal because that the laser beam has suffered severe attenuation. The intensity changes significantly with increasing time and this can be explained on the basis of increased free path of the beam and decreased photons 


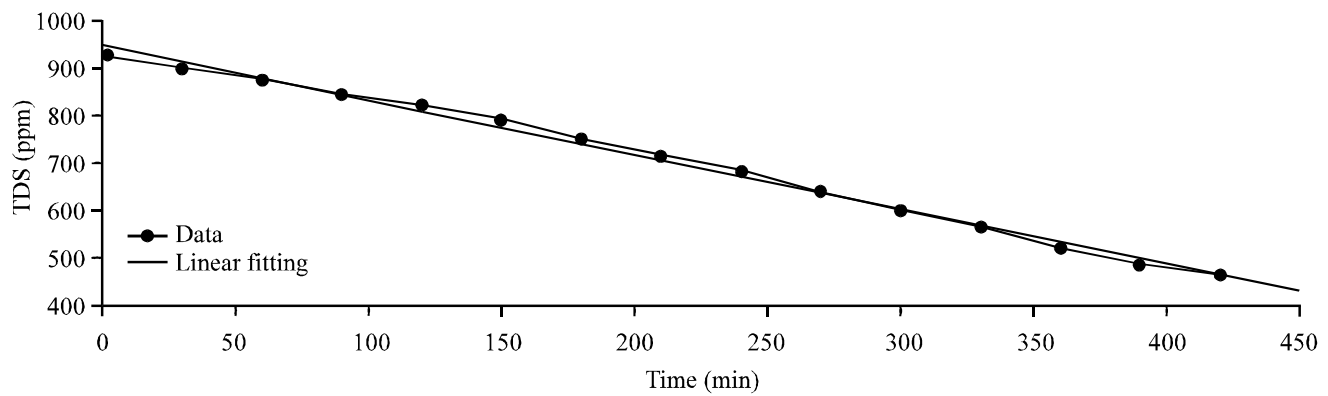

Fig. 6: Relationship between time of deposition and TDS

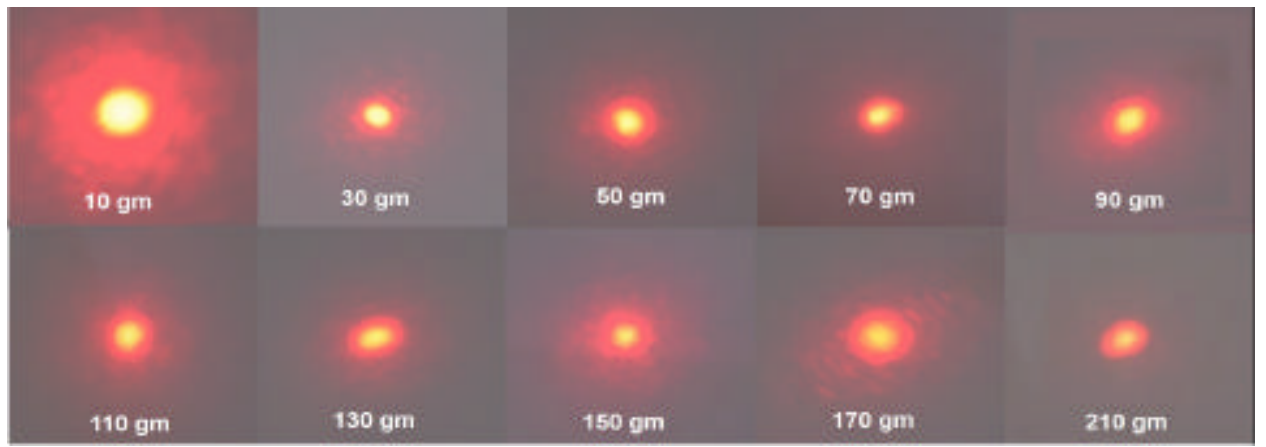

Fig. 7: Image of laser spots after passing through distilled water add to it amounts of salt

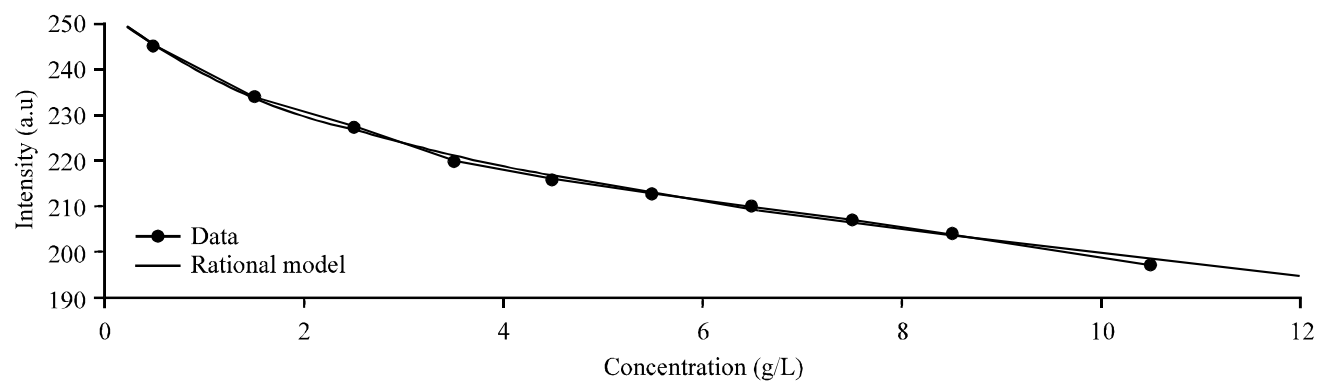

Fig. 8: Relationship between concentration and intensity

Table 1: Resulted data of Tigris River water and their fitting data

\begin{tabular}{lcccc}
\hline Time (min) & Intensity (a.u) & Intensity (fitting) & TDS (ppm) & TDS (fitting) \\
\hline 0 & 147.8 & 148.3331 & 928 & 946.4000 \\
30 & 152.8 & 154.7564 & 899 & 912.0572 \\
60 & 160.6 & 161.1797 & 874 & 877.7144 \\
90 & 167.0 & 167.6030 & 846 & 843.3716 \\
120 & 172.6 & 174.0263 & 822 & 809.0288 \\
150 & 178.5 & 180.4496 & 790 & 774.6860 \\
180 & 188.5 & 186.8729 & 750 & 740.3432 \\
210 & 196.8 & 193.2962 & 715 & 706.0004 \\
240 & 204.6 & 199.7195 & 684 & 671.6576 \\
270 & 208.9 & 206.1428 & 641 & 637.3148 \\
300 & 213.4 & 212.5661 & 599 & 602.9720 \\
330 & 218.0 & 218.9894 & 567 & 568.6292 \\
360 & 224.9 & 225.4127 & 522 & 534.2864 \\
390 & 229.9 & 231.8360 & 489 & 499.9436 \\
420 & 235.1 & 238.2593 & 464 & 465.6008 \\
\hline
\end{tabular}

that scattered during the passage of the beam inside the basin. Where by increasing time, most of the large suspended objects will be deposited causing a decrease in intensity which often causes the scattering of the beam, there is a linear relationship between the reading of the TDS device and the time of deposition showed in Fig. 6. The greater the deposition time, the lower the reading of the device due to the deposition of the insoluble salts and the suspended objects contained in the river water.

When increasing the concentration of salt in distilled water we notice a decrease in the intensity of the beam showed in Fig. 7 and 8 and this is the result of the occurrence of attenuation and high absorption of the middle result of the fact that the distilled water medium is homogeneous visually (i.e., the coefficient of refraction is constant and does not change with the difference of location) which is the chance of occurrence a scattering is weak in the case of the presence of crystals of salt that 


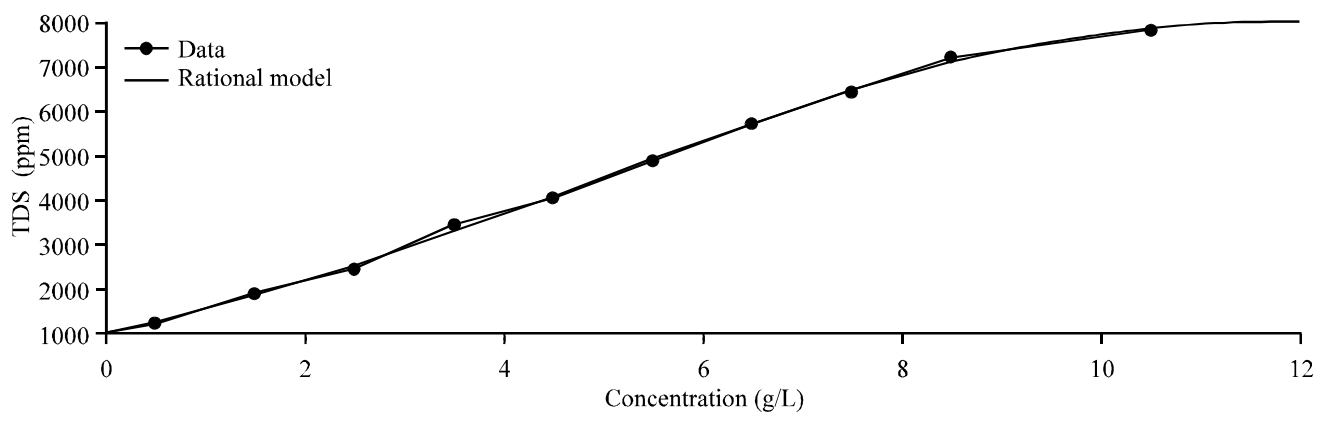

Fig. 9: A diagram showing the relationship between concentration and TDS

\begin{tabular}{|c|c|c|c|c|}
\hline $\begin{array}{l}\text { Concentration } \\
\text { (g) }\end{array}$ & $\begin{array}{l}\text { Intensity } \\
\text { (a.u) }\end{array}$ & Intensity (fitting) & $\begin{array}{l}\text { TDS } \\
(\mathrm{ppm})\end{array}$ & TDS (fitting) \\
\hline 0.5 & 245.3 & 245.431512426 & 1255 & 1282.65066815 \\
\hline 1.5 & 234.3 & 233.908895948 & 1910 & 1867.46824842 \\
\hline 2.5 & 227.2 & 226.609447623 & 2460 & 2538.70647662 \\
\hline 3.5 & 220.0 & 221.161818569 & 3410 & 3287.75659793 \\
\hline 4.5 & 216.0 & 216.706905600 & 4070 & 4094.23174196 \\
\hline 5.5 & 212.8 & 212.857397337 & 4900 & 4924.45144384 \\
\hline 6.5 & 210.0 & 209.412475496 & 5700 & 5733.09621751 \\
\hline 7.5 & 207.0 & 206.257236631 & 6430 & 6469.03306905 \\
\hline 8.5 & 204.0 & 203.320889986 & 7170 & 7084.50429447 \\
\hline$\underline{10.5}$ & 197.0 & 197.934571547 & 7810 & 7834.23705155 \\
\hline
\end{tabular}

is not completely dissolved which causes scattering in the incident beam. In addition, the most important reason lies in the amount of ions produced in the water that causes the scattering, we also notice that the general appearance of the spot did not suffer any change, i.e, there is no change in the phase of the moving beam during the medium or the distortion of the transmitted intensity which is in fact due to the optical homogeneity of the distilled water which makes it a medium of attenuation and not scattering medium (Table 2) (Fig. 9).

\section{CONCLUSION}

We can study the relationship between the concentration of liquids depend on the attenuation of laser by imaging laser spot that passes through the medium. The research shows the ability of digital image processing to extract and analysis the data instead of traditional sensors.

This system is low cost, easy and effective. The research introduced a new method to study and predict some physical properties about different media. This method is believed to give more accurate results than the TDS device because it measures the salinity ratio within a specific small area. In this method, the salinity ratio is measured within a much larger range. When tds is used to calibrate the data, the salinity ratio is measured for more than one point within the same medium. This is because the concentration is uneven for all center regions.
The equations which resulted by curve fitting criteria gives an important applications that can be used to predict any concentration of materials in same media like air and liquds. Also these equations can be modeled in available software for handle devise was built to measure the concentration in multimedia.

\section{REFERENCES}

Al-Azzawi, Z.M. and N.Z. Abdulzahra, 2014. Optical properties of tap water purity using He-Ne laser with different power density. J. Al Nahrain Univ. Sci., 17: 99-107.

Al-Rogaibah, R.S., 2009. A Brief Introduction to Laser Principles. MSc Thesis, King Fahd University of Petroleum and Minerals, Dhahran, Saudi Arabia.

Allmen, M.V. and A. Blatter, 2013. Laser-Beam Interactions with Materials: Physical Principles and Applications. 2nd Edn., Springer, Berlin, Germany, ISBN:978-3-540-59401-7, Pages: 196.

Banuelos-Cabral, E.S., J.A. Gutierrez-Robles and B. Gustavsen, 2017. Rational Fitting Techniques for the Modeling of Electric Power Components and Systems Using MATLAB Environment. In: Rational Fitting Techniques for the Modeling of Electric Power Components and Systems Using MATLAB Environment, Banuelos-Cabral, E.S., J.A. GutierrezRobles and B. Gustavsen (Eds.). Intech Open, Rijeka, Croatia, pp: 148.

Hansen, P.C., V. Pereyra and G. Scherer, 2013. Least Squares Data Fitting with Applications. Johns Hopkins University Press, Baltimore, Maryland, USA., ISBN-13:978-1-4214-0786-9, Pages: 305.

Jassim, J.M., 2014. Evaluation study of underwater laser communication system in shatt Al-Hilla-Iraq. Aust. J. Basic Applied Sci., 8: 102-106.

Zielesny, A., 2016. From Curve Fitting to Machine Learning: An Illustrative Guide to Scientific Data Analysis and Computational Intelligence. 2nd Edn., Springer, Berlin, Germany, ISBN:978-3-319-32544-6, Pages: 498. 